\title{
Intracerebral Hemorrhage, Indication for Surgical Treatment and Surgical Techniques
}

\author{
R. Reichart ${ }^{*}$ and S. Frank \\ Department of Neurosurgery, Jena University Hospital, Friedrich-Schiller-University, Erlanger Allee 101, D-07747 \\ Jena, Germany
}

\begin{abstract}
Intracerebral hemorrhage is a common disease. We give a short review of primary haemorrhages under neurosurgical aspects. Neurological symptoms depend on localisation and size of the hematoma. Only a subgroup of intracerebral haemorrhages is treated surgically. The STICH-trial tried to differentiate, in which cases the hematoma should be removed surgically. Surgical treatment could be performed by open craniotomy, endoscopic evacuation of the hematoma or CT-guided stereotaxy.
\end{abstract}

Keywords: Intracerebral hemorrhage, neurosurgical treatment, ICH.

\section{INTRODUCTION}

Intracerebral hemorrhage (ICH) is a common disease with an incidence ranging from 11 to 23 cases per 100,000 per year. Although it accounts for only 10 to $15 \%$ of all strokes, it is the most fatal stroke subtype with a mortality up to $40 \%$ [1].

Intracranial hemorrhages can be classified by anatomical or etiological aspects. According to anatomical considerations parenchymatous, subarachnoid, subdural, epidural, supra- and infratentorial hemorrhages have been found.

According to etiological aspects primary or spontaneous hemorrhages can be distinguished from secondary hemorrhages. Primary haemorrhages are spontaneous hemorrhages, which are mainly caused by arterial hypertensive diseases. Secondary hemorrhages are due to traumatic, tumorous or pharmacological causes. This review article will consider primary intracerebral hemorrhages only.

In most cases primary intracerebral hemorrhages are caused by arterial hypertension, (in up to $90 \%$ of patients) or by amyloid angiopathy. The localisation of the hematoma is associated with the etiology of hemorrhage. Hypertensive hemorrhages p.e. involve frequently basal ganglia, thalamus or the posterior fossa. The primary hemorrhages based on amyloid angiopathy may result in lobar hematomas, typically at the border of the grey and white matter. But cerebral amyloid angiopathy associated haemorrhages can also involve the subarachnoidal space or result in cerebellar hematomas $[2,3]$.

The exact etiology and pathophysiology of a primary intracerebral hematoma remain controversal. Primary intracerebral haemorrhages are caused of the rupture of small

\footnotetext{
*Address correspondence to this author at the Department of Neurosurgery, Jena University Hospital, Friedrich-Schiller-University Jena, Erlanger Allee 101, D-07747 Jena, Germany; Tel: ++49 +3641 9323023; Fax: ++49 +3641 9323021; E-mail: Rupert.Reichart@med.uni-jena.de
}

arterioles, in most cases associated with longterm arterial hypertension. Spontaneous rupture of these blood vessels due to reduced vessels elasticity and increased susceptibility [4]. Cerebral amyloid angiopathy is a common cause of ICH in the elderly, which is not associated with arterial hypertension desease [4].

While expanding, the intracerebral hematoma takes the path of lowest resistance and spreads along the neuronal fibres. Supratentorial located ICH causes rising of the intracranial pressure if its volume is larger than $60 \mathrm{ml}$ or more in the presence of an atrophy of the brain. Finally, rising tissue pressure and hemostasis finally stop bleeding. Raised tissue-pressure next to ICH causes danger of ischemia in this area, which leads to a cytotoxic brain edema within 24 to 48 hours, enlarging during the next days. These mechanisms result into a secondary raised intracranial pressure, which causes secondary neurological damages and needs further treatment.

\section{SYMPTOMS}

Depending on localisation and volume of the hematoma, ICH may cause different neurological deficits. Like in ischemic insults, in ICH neurological symptoms are developed within minutes to hours. Symptoms of ICH can be divided into general manifestation and manifestations due to the localisation of hematoma. Normally, severe headaches are described sometimes in combination with vomiting, whereas an alteration in level of consciousness also could occur. Symptoms of lobar ICH are associated with the affected cerebral lobe, so homonyme hemianopsia, paresis of arm or leg, or aphasia are observed. Small ICH of basal ganglia could occur without any symptoms, but larger ICH in this region leads to sensomotoric contralateral hemiparesis, sometimes in combination with aphasic disorders or homonymous hemianopsia, when the hematoma extends posteriorly and involves optic radiation. In ICH of Pons severe neurological deficits are observed like coma, disorders of pupillomotoric, abnormal flexions or extensions of extremities. Cerebellar ICH typically causes nausea, 
vomiting, and dizziness. Hydrocephalus may occur, if circulation pathways of CSF are obstructed, leading to an alteration in level of consciousness. Larger cerebellar haemorrhages could lead to brain stem compression. In these cases an alteration in level of consciousness, tetraparesis or paresis of cranial nerves could be observed. Hematomas located exclusively intraventriculary usually cause headaches only, although a secondary hydrocephalus can lead to unconsciousness.

\section{DIAGNOSIS}

If $\mathrm{ICH}$ is suspected, cerebral imaging is obligatory. Cranial CT has a good sensitivity (more than 95\%), however there are cases in which hemorrhage does not appear in cranial CT but are detected in MR-imaging (with T2* and protonweight sequences) [5]. Fiebach even reports of a sensitivity up to $100 \%$ when using MR-imaging [6]. Since computed Imaging is more available und easier in use an unenhanced CT-scan is the first choice for diagnostic examination. In young patients or patients with no preexisting arterial hypertension further diagnostic imaging is advisable, using MR-angiography, CT-angiography, or conventional digital substraction angiography [7].

\section{THERAPY}

It is point of dicussion for more than 100 years whether ICH should be evacuated surgically. Surgeons noticed that the prognosis for patients still remained poor, independently whether they were surgically treated or not. Up to $30 \%$ of all patients suffering from ICH die within 30 days after stroke, and many patients surviving ICH remain in a severe affected vegetative state. Thus the outcome of a large part of patients is still unsatisfactory. Multiple attempts have failed to find objective criteria to decide, whether surgery is useful or not in a single case, although the surgical techniques have been improved during the recent years.

These questions should be finally answered by the STICH-trial (International Surgical Trial in Intracerebral Hemorrhage), a large randomized multicentric trial [8]. Overall 1033 patients with spontaneous supratentorial ICH have been enrolled within 72 hours after stroke. If there was uncertainty of the advantage of surgical or medical treatment, the patient has been randomised. For enrolling a patient, ICH should have a diameter more than $2 \mathrm{~cm}$, and GCS should be better than 5. Hemorrhages caused by a vascular abnormality, brain tumor or trauma were excluded, as well as ICH located in the cerebellum or in the brain stem. When randomized to the surgical treatment in about $75 \%$ craniotomy was done. The trial showed that there are no significant differences between surgical and non surgical treated groups. A good outcome was observed in $26 \%$ of the surgical und $24 \%$ of the medical treated group. Even the mortality after 6 months was nearly identical: $36 \%$ versus $37 \%$ respectively. Furthermore the trial showed that poor initial GCS (less than 9) is associated with poor outcome regardless of surgical or nonsurgical treatment.

The results of STICH are still discussed and interpreted in different ways. Two main points of view are criticized, which are undermining the results of this trial. First, $26 \%$ of initial medical treated patients shifted to surgical treatment because of secondary detoriation (worsening of consciousness, new neurological symptoms or rebleeding). Second, the including criteria have been set according to the clinical uncertainty principle, so due to multicentric trial (83 hospitals in 27 countries) is was not possible to give a definitely statement for all patients.

After all the STICH-trial showed that patients with surficial hemorrhages (distance to cortex surface less than $1 \mathrm{~cm}$ ) seem to benefit from surgery. These results should be verified now by the STICH-2-trial.

Only in a small part of ICH there is uncertainty whether surgery should be done or not.

Small hemorrhages especially of the basal ganglias with small or absent neurological deficits should not be treated surgically. (Fig. 2). But also in large hematomas damaging a whole hemisphere, surgery is not advisable, especially in old patients in a poor neurological state. Still there does not exist any evidence by clinical trials, because randomising in this case seems to be not acceptable for ethical reasons.

Patients suffering from medium-sized lobar ICH should undergo surgery, particulary if GCS is below 7. (Fig. 1). Those showing small initial hematomas and detoriating in clinical course, should also be treated surgically.
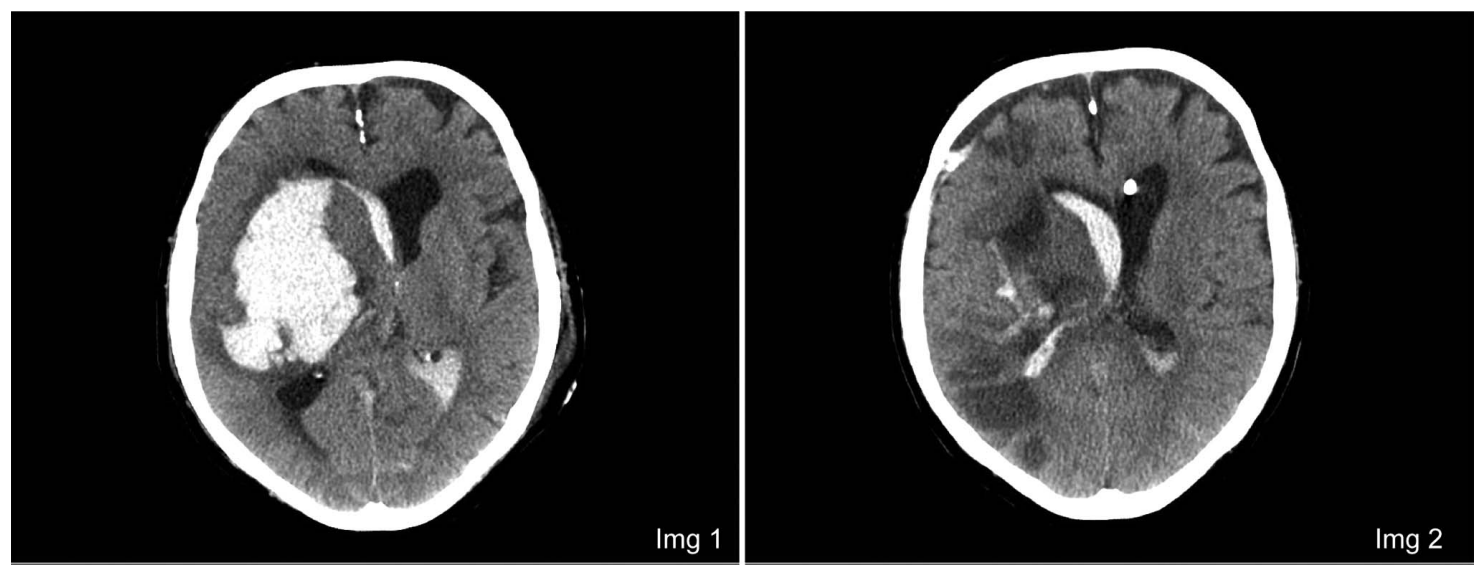

Fig. (1). Basal ganglia ICH treated surgically. Basal ganglia ICH on the right side, showing a mass effect and resulting into upper herniation. Image 1 before surgical evacuation via open craniotomy, image 24 days after surgery, presenting now an infarction of A. cerebri media right side. 

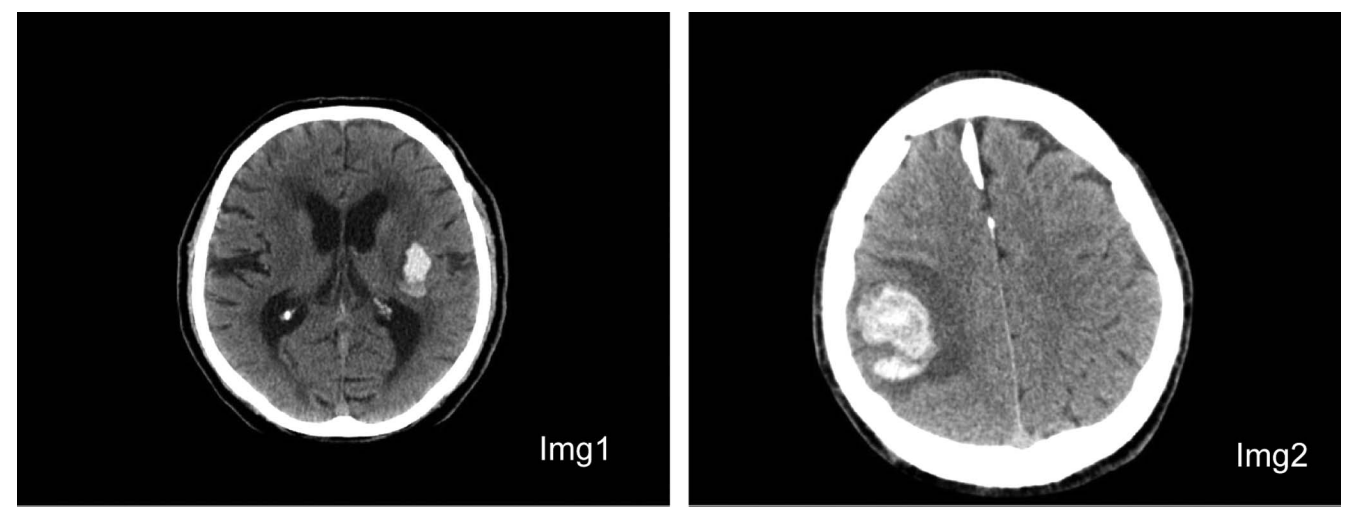

Fig. (2). Small ICH treated non-surgically. Small ICH of the basal ganglia (Img1), needing no further surgical treatment. Lobar ICH with surrounding edema (Img2), also medical treated.

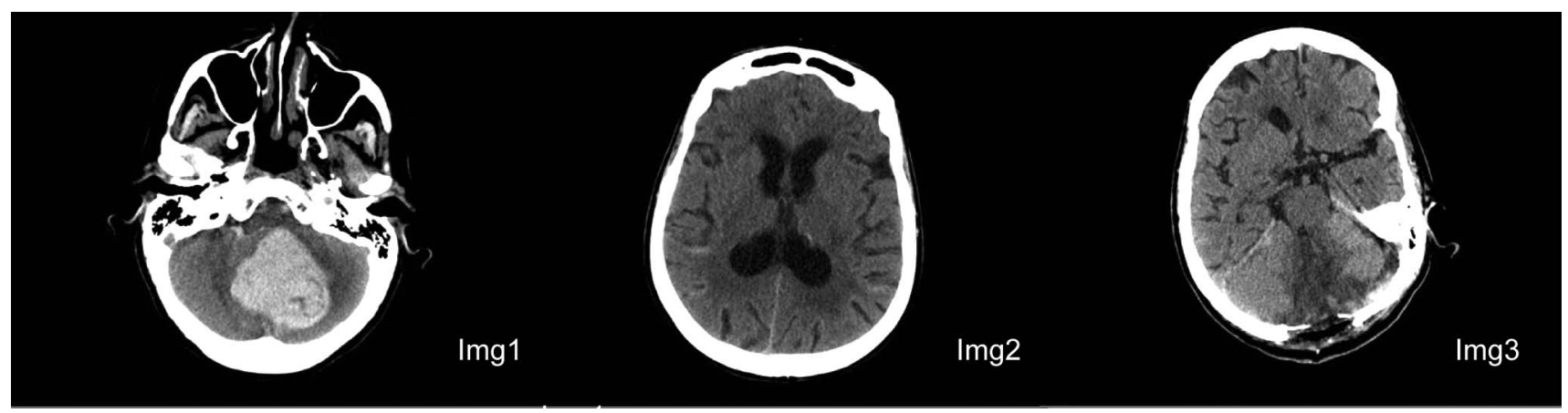

Fig. (3). Cerebellar ICH treated surgically. Cerebellar ICH with compression of the brain stem (Img1), developing a hydrocephalus through obstructing the CSF pathways (Img2). Dekompression of the posterior fossa and evacuation of the hematoma have been done immediately. (Img3).

Indication to surgery should be expressed generously if cerebellar ICH is causing disorders in circulation of the CSF or ICH is affecting the brain stem by a mass effect. (Clinical signs are reduction of consciousness, swallowing disorders, or abducens palsy). (Fig. 3). In cases of small cerebellar hemorrhages and disorders in circulation of the CSF only a temporary external ventricular drain should be implanted, but the hematoma itself should not be evacuated.

\section{SURGICAL THERAPIES}

\section{Open Craniotomy and Evacuation of the Hematoma}

In principle craniotomy should be done in a way, that the hematoma can be reached on the shortest path as possible avoiding further injury to eloquent brain-areas. After smallest possible incision, the cortex is forced apart with self-retaining spatula and the hematoma cavity is localised. Parts of the hematomas are removed with forceps, while paying attention to avoiding new bleeding in margin zones of the hematoma. The use of an operation microscope is advantageous for adequate hemostasis and satisfactory hematoma removal.

\section{Endoscopic Evacuation of the Hematoma}

Endoscopic guided evacuation of the hematoma with coagulation by neodym-laser is propagated in the end of the 1980ies [9]. Surgery can be performed through a single burr hole. While evacuating the hematoma the direction of the endoscope is changed to inspect all directions of the hematoma cavity for bleeding vessels, which could be coagulated. Some authors described a better neurological outcome compared to an open craniotomy or CT guided stereotaxy in small groups, but further randomized trials are still missing $[10,11]$.

\section{Computer Tomography (CT) - Guided Stereotaxy}

In 1960ies stereotactic aspiration of deep localised ICH has been developed as influenced by the development and introduction of computed imaging. Since coagulated hematomas are difficult to aspirate, urokinase may be injected into the hematoma cavity for residual hematoma lyses and to enable draining the hematoma. Using this procedure an inspection of the hematoma cavity is not possible. Because of a poor bleeding control there is a higher risk of rebleeding compared to both other surgical techniques. CT-guided stereotaxy is used in some ICH, but evidenced experience is missing to verify the effictiveness of this procedure [10-12].

\section{SUMMARY AND CONCLUSION}

Primary intracerebral hemorrhage is a common disease. Although surgical technique has been improved in recent time the prognosis for patients suffering from ICH still remains poor.

In large hemorrhages including the brain stem or the thalamus surgery is not advisable.

In putaminal or lobar ICH the clinical state of the patient should be taken into consideration. Consciounent patients with hematomas between 30 and $50 \mathrm{ml}$ should undergo 
medical treatment. Evacuating of larger hematomas in unconsciounent patients could be lifesaving. Especially in old unconsciounent patients showing signs of affection of the brain stem, the prognosis seems to be hopeless, thus surgery is not advisable. If secondary neurological detoriation occurs and the volume of hematoma has a size of 50 to $60 \mathrm{ml}$, open craniotomy and evacuating of the hematoma could rescue from death. In all cases the clinical state and the personal history of the patient has to be taken into consideration. A very poor neurological or functional outcome should be avoided.

Cerebellar hematomas are a special subtype, showing a good clinical outcome if the initial clinical state is good. In this case decompression should be done immediately to avoid compression of the brain stem.

\section{REFERENCES}

[1] Kirkman MA, Mahattanakul W, Gregson BA, Mendelow AD. The effect of the result of the STICH trial on the management of spontaneous supratentorial intracerebral haemorrhage in Newcastle. Br J Neurosurg 2008; 22(6): 739-46.

[2] Kaufmann HH. Intracerebral Haematomas. Raven Press: New York, 1992.

[3] Masuda J, Tanaka K, Ueda K, Omae T. Autopsy study of incidence and distribution of cerebral amyloid angiopathy in Hisayama, Japan. Stroke 1988; 19: 205-10.
[4] Elijovich L, Pratik VP, Hemphill JC. Intracerebral Hemorrhage. Semin Neurol 2008; 28: 657-67.

[5] Kidwell CS, Chalela JA, Saver JL, et al. Comparison of MRI and CT for detection of acute intracerebral hemorrhage. JAMA 2004; 292: 1823-30.

[6] Fiebach JB, Schellinger PD, Gass A, et al. Stroke magnetic resonance imaging is accurate in hyperacute intracerebral hemorrhage: a multicenter study on the validity of stroke imaging. Stroke 2004; 35: 502-6.

[7] van Straaten EC, Scheltens P, Barkhof F. MRT and CT in the diagnosis of vascular dementia. J Neurol Sci 2004; 226: 9-12.

[8] Mendelow AD, Gregson BA, Fernandes HM, et al., STICH Investigators. Early surgery versus conservative treatment in patients with spontaneous supratentorial intracerebral haematomas in the International Surgical Trial in Intracerebral Haemorrhage (STICH): a randomized trial. Lancet 2005; 365(9457): 387-97.

[9] Auer LM, Holzer P, Ascher PW, Heppner F. Endoscopic neurosurgery. Acta Neurochir (Wien) 1988; 90(1-2): 1-14.

[10] Nishihara T, Morita A, Teraoka A, Kirino TK. Endoscopy-guided removal of spontaneous intracerebral hemorrhage: comparison with computer tomography-guided stereotactic evacuation. Childs Nerv Syst 2007; 23: 677-83.

[11] Cho D, Chen C, Chang C, Lee W, Tso M. Endoscopic surgery for spontaneous basal ganglia hemorrhage: comparing endoscopic surgery, stereotactic aspiration, and craniotomy in noncomatouse patients. Surg Neurol 2006; 65: 547-56.

[12] Matsumoto K, Hondo H, Farided C. Stereotaxic evacuation of hypertensive intracerebral haematomas. J Neurosurg 1984; 61: 4408 .

(C) Reichart and Frank; Licensee Bentham Open.

This is an open access article licensed under the terms of the Creative Commons Attribution Non-Commercial License (http://creativecommons.org/licenses/ by-nc/3.0/) which permits unrestricted, non-commercial use, distribution and reproduction in any medium, provided the work is properly cited. 